\title{
Respiratory tract mucous membrane microecology and asthma
}

\author{
Xingyuan Chen, Chen Qiu \\ Department of Respiratory and Critical Care Medicine, Shenzhen Institute of Respiratory Diseases, Shenzhen People's Hospital, Shenzhen 518020, \\ China \\ Contributions: (I) Conception and design: C Qiu; (II) Administrative support: None; (III) Provision of study materials or patients: None; (IV) \\ Collection and assembly of data: X Chen; (V) Data analysis and interpretation: All authors; (VI) Manuscript writing: All authors; (VII) Final approval \\ of manuscript: All authors. \\ Correspondence to: Chen Qiu, PhD. Department of Respiratory and Critical Care Medicine, Shenzhen People's Hospital, Shenzhen 518020, China. \\ Email: szchester@163.com.
}

\begin{abstract}
According to the world health organization, the increasing incidence of asthma is placing a heavy burden on the social economy. Its high rate of disability and mortality has become a serious social and public health problem. Asthma is a heterogeneous disease in which genetic polymorphism interacts with environmental factors. Because the pathogenesis of asthma is not completely clear, there is no specific treatment. In 2010, 16S rRNA gene sequencing showed that lungs have many different microbial communities in both healthy and sick states. These microbial communities and respiratory mucosa constitute the respiratory mucosal microecology. When the respiratory mucosal microecology changes, it can play a key role in the occurrence and development of asthma and other respiratory diseases by regulating the immune mechanism. This paper reviews the latest research results in this field, and tries to explore the effects of changes in respiratory mucosal microecology on the pathogenesis of asthma, so as to provide new methods for early diagnosis, treatment and prevention of asthma.
\end{abstract}

Keywords: Respiratory mucosal microecology; asthma; immunity

Submitted Aug 06, 2019. Accepted for publication Aug 20, 2019.

doi: 10.21037/atm.2019.09.06

View this article at: http://dx.doi.org/10.21037/atm.2019.09.06

\section{Introduction}

Due to the limitations of traditional pathogen culture, it has long been considered that in healthy people, the lower respiratory tract is sterile. In 2010, however, metagenomics $16 \mathrm{~S}$ rRNA gene sequencing-based research revealed that in both healthy and diseased states, there are many different microbial communities in the lungs (1). As such, the respiratory mucosal microecology is composed of different microbial communities in different tissues, structures, and physical environments of the respiratory tract. A change in any component may tip the balance, which may lead to pathophysiological changes in the respiratory tract and ultimately culminate in the occurrence and development of asthma (1).

\section{Microecology in the respiratory tract and immunity}

Respiratory tract microecology is an intact system composed of microbial flora and the tissue, anatomy, and physical environment of the respiratory tract, among which there is tremendous exchange of information, energy, and substances. Among them, the respiratory mucosa is an important part of respiratory microecology, which mediates contact recognition with foreign antigens (e.g., symbionts, pathogens) and generates immune tolerance or immune clearance. In other words, it is a membrane immune barrier for a host to resist foreign pathogens. The balance between microbial communities and the host can become compromised, resulting in a pathological state that 
is referred to as dysbiosis (2).

The key components of the mucosal immune barrier are immune cells (e.g., T lymphocytes, B lymphocytes, natural killer (NK) cells, bronchial epithelial cells, and dendritic cells) and immune factors (e.g., secretory IgA) (3). $\mathrm{T}$ cells are involved in cellular immunity, whereas B cells differentiate into plasma cells, secrete targeted antibodies and present antigens to participate in humoral immune response (4). Dendritic cells locate on the surface of the mucous membrane and are able to sense external stimuli. Although the number of dendritic cells is relatively small, upon activation, they can cooperate with $\mathrm{T}$ and $\mathrm{B}$ cells to regulate immune processes (5). NK cells recognize antigens through leukocyte functional-associated antigen-1 (LFA-1) and adhesion molecule- 1 and subsequently release perforin and other cytotoxic factors, whereby target pathogens are killed (6). Treg cells maintain mucous immune tolerance, whereas IgA plays a protective role by preventing pathogens from adhering to the epithelium (7). Symbiotic bacteria and the aforementioned cells are the major constituents that form the mucosal immune barrier. They coexist, promote each other, and restrict each other, whereby they maintain homeostasis of the respiratory tract $(8,9)$.

The mucous membrane of the host provides nutritional support for symbiotic bacteria in the respiratory tract, which in turn promotes the formation and development of the mucosal immune system. Their collaboration helps the host resist invasion from pathogenic bacteria. A lack of exposure to symbiotic bacteria in the early stage of life can lead to irreversible damage to the host and induce immune deviation (10). Certain symbiotic bacteria can inhibit the secretion of Th2 cytokines including interleukin (IL)-4, IL-5, IL-9, and IgE, decrease the infiltration of inflammatory cells, induce IgA generation, and enhance the numbers of CD4 and CD8 T cells, which reduce the colonization of foreign pathogens and corresponding inflammatory responses and damage to the host. Bacteroides fragilis or segmented filamentous bacteria (SFB) can promote the transformation of $\mathrm{T}$ cells to Th1/Th2 cells or Th17 cells and augment the function of Treg cells. When the host immunity shifts towards Th1 cells, the immune inflammatory response induced by Th2 cells is reduced. Moreover, Treg cells can also inhibit autoimmunity, thereby maintaining homeostasis of the mucous barrier (11). Clostridia and other symbiotic bacteria are involved in the generation and development of Treg cells and NK cells and maintain intact immune cells (12). Novak virus can cooperate with Bacteroides to stimulate the differentiation of lymphocytes and minimize the damage caused by interferon $\lambda$ (13). Furthermore, phages can kill pathogenic bacteria and provide an additional defense mechanism to protect the mucous membrane barrier from damage (14).

In conclusion, symbiotic bacteria promote the formation and development of the mucosal immune barrier, regulate immune responses to pathogens, and maintain homeostasis. Furthermore, the mucous membrane immune barrier provides a shelter for symbiotic bacteria and modulates their composition. Although there is a preliminary understanding of respiratory microecology, many questions remain to be studied further.

\section{Respiratory tract microecology under abnormal circumstances and its influence on immunity}

External environmental fluctuations, antibiotic use, dietary alteration, and physiological and psychological changes can all deplete symbiotic bacteria and enrich pathogenic bacteria in the respiratory tract. This results in local dysbiosis even in the absence of any cellular, structural, and environmental changes in the respiratory tract and subsequently leads to a variety of clinical symptoms in the human body.

The upper respiratory tract of healthy individuals is colonized mainly by bacteria from 5 phyla: Firmicutes (53.139\%), Bacteroides, Proteobacteria, Actinobacteria and Fusobacterium (15). In addition, parvovirus and adenovirus are the viral symbionts in the respiratory tract. Studies have suggested that Candida, Cladosporium and unknown Saccharomyces species are common symbiotic fungi in healthy individuals. When the internal and external environments of the host change, the density and quantity of the aforementioned resident microbes decrease, whereas a large number of pathogenic agents (Haemophilus influenzae, Streptococcus pneumoniae, influenza virus, respiratory syncytial virus, coronavirus and Aspergillus spp., etc.) propagate and adhere to epithelial surface receptors, thus initiating a plethora of immune reactions that eventually lead to the occurrence of diseases (16).

Under abnormal circumstances, the changes in epithelial cells, immune cells and other cells in the respiratory tract will also affect the respiratory track microecology: (I) epithelial cells are the key components of inflammatory responses and the source of pro-inflammatory cytokines. Pathogenic substances can destroy the integrity of epithelial cells and lead to the death and exfoliation of epithelial cells. Damaged epithelial cells lead to mucociliary dysfunction and increased mucus viscosity, which prevent pathogen 
removal and even facilitate their invasion into submucosal tissue. Toll-like receptors in residual epithelial cells trigger intracellular signals, activate nuclear transcription factors and secrete a variety of pro-inflammatory cytokines to regulate airway inflammation. In addition, epithelial damage can trigger the influx of major eosinophilic granulocytes or neutrophils into the airway and aggravate disease progression (17); (II) when dysbiosis occurs, neutrophils accumulate rapidly at the inflammatory site and secrete myeloperoxidase, proteases and defensin before they remove the pathogen directly or indirectly. Neutrophils also exhibit chemotactic responses towards other neutrophils, monocytes, and immature dendritic cells (18); (III) After recognizing a pathogen, alveolar macrophages can capture and kill the antigen. When an infection is serious, alveolar macrophages can recruit neutrophils through cell-tocell signal transduction, promote respiratory burst and phagocytosis, and, ultimately, eliminate the pathogen (19); (IV) after recognizing antigens through their respective receptors, T-cell receptor (TCR) or B-cell receptor (BCR), T cells or B cells express a large number of surface molecules and receptors and produce effector molecules and antibodies, thereby playing an antibacterial role (20); (V) dendritic cells are professional pathogenic antigen presenting cells that can ingest processed antigens and induce an immune response (21); (VI) B cells can produce $\operatorname{IgA}, \operatorname{IgG}, \operatorname{IgE}$ and $\operatorname{IgM}$ and participate in humoral immunity. These antibodies can block the cellular adhesion of pathogenic bacteria, neutralize exotoxin, activate the complement system, initiate complement cytotoxicity, and mediate the killing of target cells by multiple immune cells (22-24); these activities contribute to the reversal of dysbiosis in the respiratory tract.

\section{Effects of abnormal mucosal microecology on asthma}

Considering the anatomy and physical environment of the respiratory tract, dysbiosis resulting from alterations in microbiota and immune cells may be a more plausible cause of asthma, but the pathogenic mechanism is not fully understood. Epidemiological studies have revealed that microbial exposure, infection, microbial flora composition, and immune cell changes are closely associated with the occurrence of asthma.

A study based on 16S rRNA gene sequencing analysis of induced sputum showed that in the lower respiratory tract, patients with asthma exhibited an increased abundance of Proteobacteria as well as increased microbial diversity, whereas healthy controls exhibited Bacteroides and Actinobacteria enrichment (25). In addition, it was reported that there was a significant positive correlation between airway responsiveness and the relative abundances of Comamonadaceae, Sphingomonadaceae and Oxalobacteraceae (25). The respiratory tract of patients with severe asthma is characterized mainly by the presence of Haemophilus influenzae, Pseudomonas aeruginosa and Staphylococcus aureus, which is different from the respiratory tract of mild asthma patients. Upon onset of acute asthma, Streptococcus pneumoniae, Streptococcus pyogenes and Moraxella catarrhalis are the dominant bacteria in respiratory tract (26). In addition, viruses also play an important role in the pathogenesis of asthma. Among children under 6 years old with wheezing symptoms, the risk of asthma induced by rhinovirus was significantly increased, which was followed by respiratory syncytial virus (27). Heymann et al. reported that the viral load in patients with asthma was higher than that in healthy individuals (28). Gore et al. demonstrated that antifungal drugs can change the microbial communities colonized in the respiratory tract and increase asthma severity (29). The aforementioned findings collectively indicate that dysbiosis or changes in respiratory tract flora is involved in the occurrence of asthma. The main pathogenic mechanisms are as follows: (I) the immune function of the respiratory mucosa is affected by congenital or acquired routes. For example, cell membrane glycophospholipids of Sphingomonadaceae can be recognized by invariant NK T cells, which activate Th2 cells to induce the expression of IL-4 and IL-13, thereby aggravating inflammatory responses and leading to airway hyperresponsiveness $(26,30)$; (II) dysbiosis may affect the function of the respiratory mucosal barrier. For example, human rhinovirus infection can inhibit epithelial cell apoptosis and increase inflammatory cytokines production, which in turn leads to delayed epithelial cell repair in patients with asthma; (III) human rhinovirus infection can inhibit epithelial cell apoptosis and increase inflammatory cytokines production, which subsequently leads to epithelial cell repair in patients with asthma. Administration of IFN- $\beta$ was shown to restore epithelial cell apoptosis, inhibit viral replication and improve airway epithelial cell repair in patients with asthma (31); (IV) the microorganisms residing in respiratory tract and their metabolites directly or indirectly influence the occurrence of diseases. For example, short-chain fatty acids (SCFAs) have a close association with asthma risk. Through activating $\mathrm{G}$ protein-coupled 
receptor signaling, SCFAs can inhibit histone deacetylase and thereby control gene transcription, regulate the $\mathrm{T}$ cell development and augmenting IL-10 expression. As such, SCFAs play an important role in the prevention of asthma and inflammation (32); (V) the respiratory tract microbiota can influence drug metabolism and thus interfere with disease treatments. For example, Haemophilus parainfluenzae activates toll-like receptor 4 and subsequently activates transforming growth factor $\beta$-associated kinase-1, which induces the transcription of inflammatory factors (e.g., IL-8) and production of mitogen-activated kinase phosphatase 1 and ultimately results in asthmatic corticosteroid resistance (33). However, more studies are needed to elucidate which factors contribute to repeated episodes of asthma and poor management of breathing: insufficient immune function of the patients, increased abundance of pathogenic bacteria, or insensitivity to hormones, relaxants and other drugs.

The gut-lung axis has been shown to mediate the occurrence and development of asthma (34). Both antifungal drugs and candida colonization in the intestinal tract induce airway inflammation. Another study demonstrated that when prostaglandin E2 produced by intestinal candida reaches the lungs, it signals, through the E-prostanoid 2 receptor, an increase in intracellular levels of cyclic AMP, thereby suppressing phagocytosis by alveolar macrophages and altering the development of allergic airway diseases such as asthma (35).

The "hygiene hypothesis" suggests that children who live in a rich microbial environment at an age of 0 or 1 year old have reduced incidences of allergic diseases such as asthma. The possible mechanism by which this reduction occurs is that environmental microorganisms can "educate" and modulate the microbial community of the respiratory tract, induce a shift in the immune response to Th1 to reduce the inflammatory response, and inhibit the Th2-mediated destructive immune response, thereby preventing the occurrence of asthma (36).

\section{Microecological intervention and treatment of asthma}

Restoration and adjustment of microecological balance is a new approach for the prevention and treatment of respiratory infectious diseases. Microecological balance can be maintained by modulating the microbiota to affect immune responses.

At present, common microbiota preparations include probiotics and vaccines. Probiotics contain normal human symbionts such as Streptomyces faecalis, vitamins, and trace elements, which can be taken orally to regulate host immune responses and prevent and treat chronic respiratory diseases (37). The vaccines can be administered at any mucous membrane site to affect systemic mucosal immune responses through the common mucosal immune system (38). Although microbiota preparations have broad prospects of application, when using them for the treatment of respiratory diseases, it is still important to consider issues of safety (such as patients with hypoimmunity), tolerance, immune bias, and over immunity, among others.

Although studies have shown that dietary fiber can lead to similar changes in the intestinal and pulmonary microecological environment, it remains unclear as to whether the phenomenon stems from dietary change-based microbiota alterations or from immune response-based therapy. In addition, although the presence of symbiotic bacteria in juveniles has been demonstrated to be related to lung diseases, so far only a few studies have reported that probiotics can reduce the incidence of asthma by modulating gut microbiota. More research is needed to examine the efficacy of microflora preparations in managing dysbiosis and preventing and treating asthma.

Taken together, individual microorganisms in the respiratory tract have unique characteristics, some of which are closely related to the occurrence and development of asthma and dysbiosis of the respiratory mucosa (Figure 1). The advancement of microecological research generates new approaches for the prevention, diagnosis, and treatment of asthma. In addition, determining the type, severity, drug resistance, and prognosis of asthma according to different respiratory tract microbiota is a future research direction. The study of respiratory tract microecology is helpful for further understanding the occurrence and development of asthma and lays a basis for asthma prevention and treatment. 
Environment change: antibiotics, delivery way, dietary habit, lactation way, living environment......
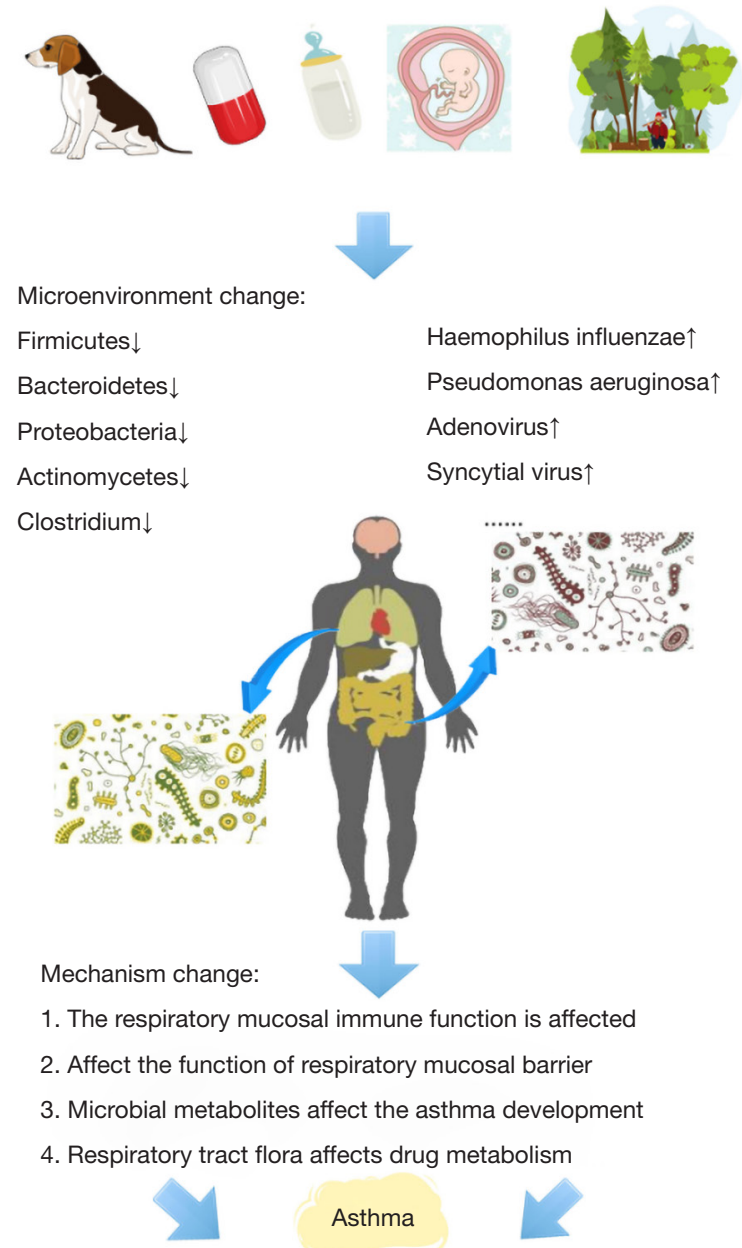

Figure 1 Overview of potential microbial influences on asthma.

\section{Acknowledgments}

None.

\section{Footnote}

Conflicts of Interest: The authors have no conflicts of interest to declare.

Ethical Statement: The authors are accountable for all aspects of the work in ensuring that questions related to the accuracy or integrity of any part of the work are appropriately investigated and resolved.

\section{References}

1. Hilty M, Burke C, Pedro H, et al. Disordered microbial communities in asthmatic airways. PLoS One 2010;5:e8578.

2. Dickson RP, Martinez FJ, Huffnagle GB. The role of the microbiome in exacerbations of chronic lung diseases. Lancet 2014;384:691-702.

3. van Unen V, Li N, Molendijk I, et al. Mass Cytometry of the Human Mucosal Immune System Identifies Tissueand Disease-Associated Immune Subsets. Immunity 2016;44:1227-39.

4. Moulton VR, Tsokos GC. T cell signaling abnormalities contribute to aberrant immune cell function and autoimmunity. J Clin Invest 2015;125:2220-7.

5. Moll H, Flohé S, Röllinghoff $M$. Dendritic cells in Leishmania major-immune mice harbor persistent parasites and mediate an antigen-specific $\mathrm{T}$ cell immune response. Eur J Immunol 1995;25:693-9.

6. Jeong JU, Uong TNT, Chung WK, et al. Effect of irradiation-induced intercellular adhesion molecule-1 expression on natural killer cell-mediated cytotoxicity toward human cancer cells. Cytotherapy 2018;20:715-27.

7. Feng T, Elson CO, Cong Y. Treg cell-IgA axis in maintenance of host immune homeostasis with microbiota. Int Immunopharmacol 2011;11:589-92.

8. Krysko O, Holtappels G, Zhang N, et al. Alternatively activated macrophages and impaired phagocytosis of $\mathrm{S}$. aureus in chronic rhinosinusitis. Allergy 2011;66:396-403.

9. Prussin C, Metcalfe DD. 4. IgE, mast cells, basophils, and eosinophils. J Allergy Clin Immunol 2003;111:S486-94.

10. Strachan DP. Hay fever, hygiene, and household size. BMJ 1989;299:1259-60.

11. Telesford KM, Yan W, Ochoa-Reparaz J, et al. A commensal symbiotic factor derived from Bacteroides fragilis promotes human CD39(+)Foxp3(+) T cells and Treg function. Gut Microbes 2015;6:234-42.

12. Leake I. Gut microbiota: Selecting Clostridia strains that induce TREG cells from gut microbiota. Nat Rev Gastroenterol Hepatol 2013;10:504.

13. Tomov VT, Palko O, Lau CW, et al. Differentiation and Protective Capacity of Virus-Specific CD8+ T Cells Suggest Murine Norovirus Persistence in an ImmunePrivileged Enteric Niche. Immunity 2017;47:723-38.e5.

14. Talago N. Bacteriophage interactions within the human mucosal immune system. San Diego State University. 2015.

15. Ormerod KL, Wood DL, Lachner N, et al. Genomic 
characterization of the uncultured Bacteroidales family S24-7 inhabiting the guts of homeothermic animals. Microbiome 2016;4:36.

16. Cordeiro Rde A, Bittencourt PV, Brilhante RS, et al. Species of Candida as a component of the nasal microbiota of healthy horses. Med Mycol 2013;51:731-6.

17. Tessier L, Côté O, Clark ME, et al. Impaired response of the bronchial epithelium to inflammation characterizes severe equine asthma. BMC Genomics 2017;18:708.

18. Wills-Karp M. Neutrophil ghosts worsen asthma. Sci Immunol. 2018. doi: 10.1126/sciimmunol.aau0112.

19. Dunster JL, Byrne HM, King JR. The resolution of inflammation: a mathematical model of neutrophil and macrophage interactions. Bull Math Biol 2014;76:1953-80.

20. Gohal G, McCusker C, Mazer B, et al. T-cell receptor phenotype pattern in atopic children using commercial fluorescently labeled antibodies against 21 human classspecific $\mathrm{v}$ segments for the $\operatorname{tcr} \beta$ chain $(\mathrm{v} \beta)$ of peripheral blood: a cross sectional study. Allergy Asthma Clin Immunol 2016;12:10.

21. van Helden MJ, Lambrecht BN. Dendritic cells in asthma. Curr Opin Immunol 2013;25:745-54.

22. Ayers CM, Schlosser RJ, O'Connell BP, et al. Increased presence of dendritic cells and dendritic cell chemokines in the sinus mucosa of chronic rhinosinusitis with nasal polyps and allergic fungal rhinosinusitis. Int Forum Allergy Rhinol 2011;1:296-302.

23. Perić A, Baletić N, Sotirović J, et al. Macrophage inflammatory protein-1 production and eosinophil infiltration in chronic rhinosinusitis with nasal polyps. Ann Otol Rhinol Laryngol 2015;124:266-72.

24. Yang LY, Li X, et al. V $\gamma 1^{+} \gamma \delta \mathrm{T}$ Cells Are Correlated With Increasing Expression of Eosinophil Cationic Protein and Metalloproteinase-7 in Chronic Rhinosinusitis With Nasal Polyps Inducing the Formation of Edema. Allergy Asthma Immunol Res 2017;9:142-51.

25. Huang YJ, Nelson CE, Brodie EL, et al. Airway microbiota and bronchial hyperresponsiveness in patients with suboptimally controlled asthma. J Allergy Clin Immunol 2011;127:372-381.e1-3.

26. Brarda OA, Vanella LM, Boudet RV. Anti-Staphylococcus aureus, anti-Streptococcus pneumoniae and anti-Moraxella catarrhalis specific IgE in asthmatic children. J Investig Allergol Clin Immunol 1996;6:266-9.

27. Olszak T, An D, Zeissig S, et al. Microbial exposure during early life has persistent effects on natural killer $\mathrm{T}$ cell function. Science 2012;336:489-93.

28. Heymann PW, Platts-Mills TA, Johnston SL. Role of viral infections, atopy and antiviral immunity in the etiology of wheezing exacerbations among children and young adults. Pediatr Infect Dis J 2005;24:S217-22, discussion S220-1.

29. Gore RB. The utility of antifungal agents for asthma. Curr Opin Pulm Med 2010;16:36-41.

30. Munitz A, Brandt EB, Mingler M, et al. Distinct roles for IL-13 and IL-4 via IL-13 receptor alpha1 and the type II IL-4 receptor in asthma pathogenesis. Proc Natl Acad Sci U S A 2008;105:7240-5.

31. Kicic A, Stevens PT, Sutanto EN, et al. Impaired airway epithelial cell responses from children with asthma to rhinoviral infection. Clin Exp Allergy 2016;46:1441-55.

32. Deng H, Fang Y. Anti-inflammatory gallic Acid and wedelolactone are $\mathrm{G}$ protein-coupled receptor-35 agonists. Pharmacology 2012;89:211-9.

33. Law DK, Shuel M, Bekal S, et al. Genetic detection of quinolone resistance in Haemophilus parainfluenzae: Mutations in the quinolone resistance-determining regions of gyrA and parC. Can J Infect Dis Med Microbiol 2010;21:e20-2.

34. Dickson RP, Cox MJ. The premature invocation of a 'gutlung axis' may obscure the direct effects of respiratory microbiota on pneumonia susceptibility. Gut 2016. doi: 10.1136/gutjnl-2016-311823.

35. Kim YG, Udayanga KG, Totsuka N, et al. Gut dysbiosis promotes M2 macrophage polarization and allergic airway inflammation via fungi-induced $\mathrm{PGE}_{2}$. Cell Host Microbe 2014;15:95-102.

36. McFadden JP, Thyssen JP, Basketter DA, et al. T helper cell 2 immune skewing in pregnancy/early life: chemical exposure and the development of atopic disease and allergy. Br J Dermatol 2015;172:584-91.

37. Russolillo N, Ferrero A, Vigano' L, et al. Impact of perioperative symbiotic therapy on infectious morbidity after Hpb Surgery in jaundiced patients: a randomized controlled trial. Updates Surg 2014;66:203-10.

38. Wagner K, Linde J, Krause K, et al. Tricholoma vaccinum host communication during ectomycorrhiza formation. FEMS Microbiol Ecol 2015. doi: 10.1093/femsec/fiv120.

Cite this article as: Chen X, Qiu C. Respiratory tract mucous membrane microecology and asthma. Ann Transl Med 2019;7(18):495. doi: 10.21037/atm.2019.09.06 